\title{
EXISTENCE OF ENTIRE EXPLOSIVE POSITIVE RADIAL SOLUTIONS OF QUASILINEAR ELLIPTIC SYSTEMS
}

\author{
YANG ZUODONG
}

Received 27 December 2002

\begin{abstract}
Our main purpose is to establish that entire explosive positive radial solutions exist for quasilinear elliptic systems. The main results of the present paper are new and extend previous results.
\end{abstract}

2000 Mathematics Subject Classification: 35J25, 35J60.

1. Introduction. Existence and nonexistence of solutions of the quasilinear elliptic system

$$
\begin{array}{ll}
-\operatorname{div}\left(|\nabla u|^{p-2} \nabla u\right)=f(u, v), & x \in \mathbb{R}^{N}, \\
-\operatorname{div}\left(|\nabla v|^{q-2} \nabla v\right)=g(u, v), & x \in \mathbb{R}^{N},
\end{array}
$$

have received much attention recently. See, for example, [5, 7, 9, 18, 26, 27, 28]. Problem (1.1) arises in the theory of quasiregular and quasiconformal mappings or in the study of non-Newtonian fluids. In the latter case, the pair $(p, q)$ is a characteristic of the medium. Media with $(p, q)>(2,2)$ are called dilatant fluids and those with $(p, q)<(2,2)$ are called pseudoplastics. If $(p, q)=(2,2)$, they are Newtonian fluids.

When $p=q=2$, system (1.1) becomes

$$
\begin{array}{ll}
-\triangle u=f(u, v), & x \in \mathbb{R}^{N}, \\
-\triangle v=g(u, v), & x \in \mathbb{R}^{N},
\end{array}
$$

for which the existence and the nonexistence of positive solutions have been investigated extensively. We list here, for example, [2, 4, 10, 13, 16, 17, 20, 22 , 23, 24].

Explosive solutions of the problem

$$
\begin{gathered}
\Delta u(x)=f(u(x)), \quad x \in \Omega, \\
\left.u\right|_{\partial \Omega}=\infty,
\end{gathered}
$$

where $\Omega$ is a bounded domain in $\mathbb{R}^{N}(N \geq 1)$ have been extensively studied, see $[1,6,11,12,14,25]$. 
In this paper, we study the existence of entire explosive positive solutions of the system

$$
\begin{array}{cl}
\operatorname{div}\left(|\nabla u|^{p-2} \nabla u\right)=m(|x|) v^{\alpha}, & x \in \mathbb{R}^{N}, \\
\operatorname{div}\left(|\nabla v|^{q-2} \nabla v\right)=n(|x|) u^{\beta}, & x \in \mathbb{R}^{N},
\end{array}
$$

where $\alpha>0, \beta>0, p>1$, and $q>1$. As far as the author knows, there are no results that contain existence criteria of entire explosive positive solutions to the elliptic system (1.4). Motivated by this fact, we will study mainly this problem here. When $p=q=2$, the related results have been obtained by [13]. Our theorem for existence extends the results [13].

A solution $(u, v)$ of the system

$$
\operatorname{div}\left(|\nabla u|^{p-2} \nabla u\right)=f(x, u, v), \quad \operatorname{div}\left(|\nabla v|^{q-2} \nabla v\right)=g(x, u, v)
$$

is called an explosive solution if $u(x) \rightarrow \infty$ and $v(x) \rightarrow \infty$ as $x \rightarrow \partial \Omega$ if $\Omega$ is bounded. If $\Omega$ is unbounded, then $(u, v)$ is an explosive solution of (1.5) on $\Omega$ if $u(x) \rightarrow \infty$ and $v(x) \rightarrow \infty$ as $x \rightarrow \partial \Omega$, and $u(x) \rightarrow \infty$ and $v(x) \rightarrow \infty$ as $|x| \rightarrow \infty$ within $\Omega$. In particular, when $\Omega=\mathbb{R}^{N}$, the solution $(u, v)$ is called an entire explosive solution of (1.5) and satisfies $u(x) \rightarrow \infty$ and $v(x) \rightarrow \infty$ as $|x| \rightarrow \infty$. Such problems arise in the study of the subsonic motion of a gas [21], the electric potential in some bodies [15], and Riemannian geometry [3].

2. Preliminaries. We first consider quasilinear elliptic inequalities of the form

$$
\operatorname{div}\left(|\nabla u|^{p-2} \nabla u\right) \geq m(x) f(u), \quad x \in \mathbb{R}^{N}(N \geq 2),
$$

where $p>1, \nabla u=\left(\nabla_{1} u, \ldots, \nabla_{N} u\right)$, and $m(x): \mathbb{R}^{N} \rightarrow(0, \infty), f:(0, \infty) \rightarrow(0, \infty)$ are continuous functions. A positive function $u \in C^{1}\left(\mathbb{R}^{N}\right)$ is defined to be a positive entire solution of inequality (2.1) if it satisfies inequality (2.1) at every point of $\mathbb{R}^{N}$.

Throughout the section, we make the following assumptions without further mention:

$\left(\mathrm{H}_{1}\right) f:(0, \infty) \rightarrow(0, \infty)$ is locally Lipschitz continuous and strictly increasing,

$\left(\mathrm{H}_{2}\right) f$ is superlinear in the sense that

$$
\int_{1}^{\infty}\left(\int_{0}^{u} f(s) d s\right)^{-1 / p} d u<\infty, \quad \int_{0}^{1}\left(\int_{0}^{u} f(s) d s\right)^{-1 / p} d u=\infty .
$$

An important special case of (2.1) satisfying the above hypotheses is the equality

$$
\operatorname{div}\left(|\nabla u|^{p-2} \nabla u\right)=m(x) u^{\gamma}, \quad x \in \mathbb{R}^{N},
$$

where $\gamma>p-1$. 
LEMMA 2.1 (weak comparison principle). Let $\Omega$ be a bounded domain in $\mathbb{R}^{N}(N \geq 2)$ with smooth boundary $\partial \Omega$ and $\theta:(0, \infty) \rightarrow(0, \infty)$ continuous and nondecreasing. Let $u_{1}, u_{2} \in W^{1, p}(\Omega)$ satisfy

$$
\begin{aligned}
& \int_{\Omega} \mid\left.\nabla u_{1}\right|^{p-2} \nabla u_{1} \nabla \psi d x+\int_{\Omega} \theta\left(u_{1}\right) \psi d x \\
& \leq \int_{\Omega}\left|\nabla u_{2}\right|^{p-2} \nabla u_{2} \nabla \psi d x+\int_{\Omega} \theta\left(u_{2}\right) \psi d x
\end{aligned}
$$

for all nonnegative $\psi \in W_{0}^{1, p}(\Omega)$. Then the inequality

$$
u_{1} \leq u_{2} \quad \text { on } \partial \Omega
$$

implies that

$$
u_{1} \leq u_{2} \quad \text { in } \Omega \text {. }
$$

Proof. Let $\eta=\nabla u_{1}, \eta^{\prime}=\nabla u_{2}$, and $\psi=\max \left\{u_{1}-u_{2}, 0\right\}$. Since $u_{1} \leq u_{2}$ on $\partial \Omega, \psi$ belongs to $W_{0}^{1, p}(\Omega)$. Inserting this function $\psi$ into (2.4), we have

$$
\int_{\left\{u_{1}>u_{2}\right\}}\left[\left(|\eta|^{p-2} \eta-\left|\eta^{\prime}\right|^{p-2} \eta^{\prime}\right)\left(\eta-\eta^{\prime}\right)+\left(\theta\left(u_{1}\right)-\theta\left(u_{2}\right)\right)\left(u_{1}-u_{2}\right)\right] d x \leq 0 .
$$

Since $\theta$ is nondecreasing, we know that

$$
\int_{\left\{u_{1}>u_{2}\right\}}\left[\left(|\eta|^{p-2} \eta-\left|\eta^{\prime}\right|^{p-2} \eta^{\prime}\right)\left(\eta-\eta^{\prime}\right)\right] d x \leq 0 .
$$

We suppose that $\mathfrak{R}(\mathfrak{I}):=\left\{x:|\eta(x)| \leq(\geq)\left|\eta^{\prime}(x)\right|\right\}$, then

$$
\frac{1}{4}\left|\eta-\eta^{\prime}\right| \leq\left|\eta^{\prime}+t\left(\eta-\eta^{\prime}\right)\right| \leq 1+|\eta|+\left|\eta^{\prime}\right| \quad \text { in } \mathfrak{R}(\mathfrak{J}),
$$

for all $t \in[0,1 / 4]$ (or $[3 / 4,1])$. Therefore, write $a^{i}(\eta)=|\eta|^{p-2} \eta_{i}$ and

$$
\begin{aligned}
0 & \geq \int_{\left\{u_{1}>u_{2}\right\}}\left(|\eta|^{p-2} \eta-\left|\eta^{\prime}\right|^{p-2} \eta^{\prime}\right)\left(\eta-\eta^{\prime}\right) d x \\
& =\int_{\left\{u_{1}>u_{2}\right\}} \int_{0}^{1} \sum_{0}^{N} \frac{\partial a^{i}}{\partial \eta_{j}}\left(\eta^{\prime}+t\left(\eta-\eta^{\prime}\right)\right)\left(\eta_{i}-\eta_{i}^{\prime}\right)\left(\eta_{j}-\eta_{j}^{\prime}\right) d t d x \\
& \geq \gamma_{0} \begin{cases}\int_{\left\{u_{1}>u_{2}\right\}}\left(1+|\eta|+\left|\eta^{\prime}\right|\right)^{p-2}\left|\eta-\eta^{\prime}\right|^{2} d x, & \text { if } p<2, \\
\int_{\left\{u_{1}>u_{2}\right\}}\left|\eta-\eta^{\prime}\right|^{p} d x, & \text { if } p \geq 2,\end{cases}
\end{aligned}
$$

where $\gamma_{0}>0$. This is a contradiction, which implies that Lemma 2.1 is true. 
LEMMA 2.2. Let $f(u)$ satisfy the following conditions:

(i) $f(s)$ is a single-value real continuous function defined for all real values of $s$ and there exists a positive nondecreasing continuous function $F(s)$ such that $f(s) \geq F(s)$ and

$$
\int_{0}^{\infty}\left[\int_{0}^{x} F(z) d z\right]^{-1 / p} d x<\infty
$$

(ii) $m(x) \geq \beta>0$ for $x \in \mathbb{R}^{N}$, and $u$ is a solution of

$$
\operatorname{div}\left(|\nabla u|^{p-2} \nabla u\right) \geq m(x) f(u), \quad x \in \mathbb{R}^{N}(N \geq 2),
$$

in a domain $D \subset \mathbb{R}^{N}$ and continuous on its boundary $S$. Then there exists a decreasing function $g(R)$ determined by $F(u)$ such that

$$
u(P) \leq g(R(P)) .
$$

Here $P$ denotes a point in $D$ and $R(P)$ denotes its distance from $S$. The function $g(R)$ has the limits

$$
\begin{aligned}
& g(R) \longrightarrow \infty \quad \text { as } R \longrightarrow 0, \\
& g(R) \longrightarrow-\infty \quad \text { as } R \longrightarrow \infty
\end{aligned}
$$

Proof. Each point $P \in D$ can be the centre of a sphere of radius $R(P)$ which lies in $D$. Therefore, it suffices to prove the theorem in $D$ as a sphere of radius $R$, and suppose that $u$ is defined continuously on $S$. We define a function $v$ in $D$ and $S$ as the solution of the problem

$$
\begin{gathered}
\operatorname{div}\left(|\nabla v|^{p-2} \nabla v\right)=F_{1}(v), \quad x \in D, \\
v=\alpha \quad \text { on } S .
\end{gathered}
$$

In (2.15), $F_{1}(v)=\theta F(v)$, where $F(v)$ is the function occurring in condition (i) and $\theta$ is a constant, $0<\theta<\beta$. Thus, from conditions (i) and (ii) and (2.15), we have

$$
\operatorname{div}\left(|\nabla u|^{p-2} \nabla u\right)-F_{1}(u) \geq \operatorname{div}\left(|\nabla v|^{p-2} \nabla v\right)-F_{1}(v)
$$

Moreover, $\alpha$ is a positive constant which satisfies

$$
u \leq \alpha \text { on } S .
$$

The existence and uniqueness of a positive solution $v$ of (2.15) are assured because $F_{1}$ is a nondecreasing function. In fact, the existence can be obtained by the standard variational method and the uniqueness can be obtained by an idea similar to that in the proof of Lemma 2.1 (see [8]). From (2.1), (2.15), and Lemma 2.1, we have

$$
u \leq v \text { in } D
$$


We now define a function $g(R)$ by

$$
g(R)=\lim _{\alpha \rightarrow \infty} v(P) .
$$

Then, since $v$ is an increasing function of $\alpha$, we have $v(P) \leq g(R)$ for every $\alpha$. Combining this inequality with (2.18), we obtain

$$
u(P) \leq g(R) .
$$

Inequality (2.20) is the desired inequality (2.13) of Lemma 2.2. It remains to show that $g(R)$ is finite in order that (2.20) is nontrivial and that $g(R)$ satisfies (2.14) and is a decreasing function of $R$.

To this end, we must examine $v$, the solution of (2.15). First we show that $v$ must be a function of $r$ only where $r$ denotes the distance from the centre of the sphere. We can find a positive radial solution $v(r)$ of $(2.15)$ by the variational method to the equivalent form of (2.15) in $r$ :

$$
\begin{gathered}
\left(\Phi_{p}\left(v^{\prime}\right)\right)^{\prime}+\frac{N-1}{r} \Phi_{p}\left(v^{\prime}\right)=F_{1}(v), \quad v^{\prime}(0)=0, \\
v(R)=\alpha
\end{gathered}
$$

where $\Phi_{p}(s)=|s|^{p-2} s$. The uniqueness of the positive solution $v$ of (2.15) implies that $v$ is just the radial solution $v(r)$ of problem (2.21) and (2.22).

Since $v(0)$ is a monotonic increasing function of $\alpha, \alpha$ is itself uniquely determined by $v(0)$. Let $v(0)=v_{0}$. As $v_{0}$ increases, $\alpha=v(R)$ also increases. We will show that $\alpha=v(R)$ becomes infinite for some finite value of $v_{0}$.

This value of $v_{0}$ is denoted by $\lim _{\alpha \rightarrow \infty} v_{0}$, which can be used to define the function $g(R)$ in this lemma.

It is convenient to rewrite (2.21) in the form

$$
\left(r^{N-1} \Phi_{p}\left(v^{\prime}\right)\right)^{\prime}=r^{N-1} F_{1}(v)
$$

Integrating (2.23) from 0 to $r$ yields

$$
\Phi_{p}\left(v^{\prime}\right)=r^{1-N} \int_{0}^{r} s^{N-1} F_{1}(v(s)) d s .
$$

From (2.24), we see that $v^{\prime} \geq 0$. Therefore, $v$ is a nondecreasing function, and we can obtain from (2.24) that

$$
\Phi_{p}\left(v^{\prime}\right) \leq r^{1-N}\left[F_{1}(v(r))\right] \frac{r^{N}}{N}=\frac{r}{N} F_{1}[v(r)] .
$$

Inserting (2.25) into (2.21), we have

$$
\left(\Phi_{p}\left(v^{\prime}\right)\right)^{\prime} \geq \frac{F_{1}(v)}{N} .
$$


Since $v^{\prime} \geq 0,(2.21)$ also yields $\left(\Phi_{p}\left(v^{\prime}\right)\right)^{\prime} \leq F_{1}(v)$. Combining this with (2.26) leads to

$$
F_{1}(v) \geq\left(\Phi_{p}\left(v^{\prime}\right)\right)^{\prime} \geq \frac{F_{1}(v)}{N} .
$$

We now multiply (2.27) by $v^{\prime}$ and integrate from 0 to $r$ to obtain

$$
\int_{0}^{r} F_{1}(v) v^{\prime}(s) d s \geq\left(\frac{p-1}{p}\right)\left(v^{\prime}\right)^{p} \geq \int_{0}^{r} \frac{1}{N} F_{1}(v(s)) v^{\prime}(s) d s,
$$

that is,

$$
H\left(v, v_{0}\right) \geq\left(v^{\prime}\right)^{p} \geq \frac{1}{N} H\left(v, v_{0}\right)
$$

where

$$
H\left(v, v_{0}\right)=\frac{p}{p-1} \int_{v_{0}}^{v} F_{1}(z) d z
$$

This implies

$$
H^{1 / p}\left(v, v_{0}\right) \geq v^{\prime} \geq(1 / N)^{1 / p} H^{1 / p}\left(v, v_{0}\right)
$$

Then we consider $r$ as a function of $v$, and we have

$$
\int_{v_{0}}^{v} H^{-1 / p}\left(z, v_{0}\right) d z \leq r \leq N^{1 / p} \int_{v_{0}}^{v} H^{-1 / p}\left(z, v_{0}\right) d z .
$$

By condition (i), the integral in (2.32) converges as $v$ becomes infinite when $v_{0}=0$. But then the integral also converges for any value of $v_{0}>0$. If we denote its limit by $A\left(v_{0}\right)$, letting $v \rightarrow \infty$, (2.32) yields

$$
A\left(v_{0}\right) \leq r_{\infty} \leq N^{1 / p} A\left(v_{0}\right)
$$

where

$$
A\left(v_{0}\right)=\int_{v_{0}}^{\infty} H^{-1 / p}\left(z, v_{0}\right) d z, r_{\infty}=\lim _{v \rightarrow \infty} r(v)
$$

From (2.34), we see that, for each $v_{0}, v$ becomes infinite at a finite value of $r_{\infty}$ in the range indicated in (2.33). Therefore, $r_{\infty}$ is a function of $v_{0}$ and is denoted by $r_{\infty}\left(v_{0}\right)$.

The function $r_{\infty}\left(v_{0}\right)$ is continuous and nonincreasing. If it were increasing, then two solutions corresponding to different values of $v_{0}$ would have to be equal at some value of $r$. This is impossible because a solution of (2.21) with a prescribed value on the surface of a sphere is unique. Furthermore, the integral $A\left(v_{0}\right)$ tends to $+\infty$ as $v_{0}$ tends to $-\infty$, and to zero as $v_{0}$ tends to $+\infty$. Therefore, by (2.33), $r_{\infty}\left(v_{0}\right)$ behaves in the same way. We now define $g(R):=\min \left\{v_{0} \mid\right.$ $\left.r_{\infty}\left(v_{0}\right)=R\right\}$. This function is decreasing and satisfies (2.14), so it is the desired $g(R)$ of Lemma 2.2. This completes the proof of Lemma 2.2. 
LEMMA 2.3. If $f(u)$ is nondecreasing and satisfies Lemma 2.2, then in any bounded domain $D$ there exists a solution of (2.1) which becomes infinite on $S$.

Proof. We note that, for any constant $\alpha$ and any domain $D$, there exists in $D$ a solution $u_{\alpha}$ of (2.1) which is equal to $\alpha$ on $S$ provided that $f(u)$ is nondecreasing (see [8]). Furthermore, at each point of $D, u_{\alpha}$ increases with $\alpha$. If $f(u)$ satisfies Lemma 2.2(i), then Lemma 2.2 holds, and at each point $P$ in $D$, all of the $u_{\alpha}$ are bounded above. Thus, in every closed subdomain, $u_{\alpha}$ converges uniformly to a limit $u$. This limit is also a solution of (2.1). As $P$ approaches $S, u(P)$ increases infinitely since on $S, u_{\alpha}=\alpha$ becomes infinite. Thus, $u$ is the desired solution and Lemma 2.3 is proved.

LEMmA 2.4. Suppose that $m \in C\left(\mathbb{R}^{N}\right), m(x)=m(|x|) \geq C>0$ for $x \in \mathbb{R}^{N}$, and

$$
\int_{0}^{\infty}\left(t^{1-N} \int_{0}^{t} s^{N-1} m(s) d s\right)^{1 /(p-1)} d t<\infty .
$$

Then (2.3) has an entire explosive positive radial solution if $\gamma>p-1$.

Proof. From Lemma 2.3, we have that for each $k \in \mathbb{N}$, the boundary value problem

$$
\begin{gathered}
\operatorname{div}\left(\left|\nabla v_{k}\right|^{p-2} \nabla v_{k}\right)=m(|x|) v_{k}^{\gamma}, \quad|x|<k, \\
v_{k}(x) \longrightarrow \infty \quad \text { as }|x| \longrightarrow k
\end{gathered}
$$

has a positive solution. Furthermore,

$$
v_{1} \geq v_{2} \geq \cdots \geq v_{k} \geq v_{k+1} \geq \cdots>0
$$

in $\mathbb{R}^{N}$. To prove our result, we only need to prove that

(A) there exists $w \in C\left(\mathbb{R}^{N}\right), w>0$, such that $v_{k} \geq w$ in $\mathbb{R}^{N}$ for all $k$;

(B) $v \rightarrow \infty$ as $|x| \rightarrow \infty$, where $v=\lim _{k \rightarrow \infty} v_{k}$.

To prove (A), condition (2.35) implies

$$
z(r)=C-\left(\frac{\gamma-p+1}{p-1}\right) \int_{0}^{r}\left(t^{1-N} \int_{0}^{t} s^{N-1} m(s) d s\right)^{1 /(p-1)} d t
$$

where

$$
C=\left(\frac{\gamma-p+1}{p-1}\right) \int_{0}^{\infty}\left(t^{1-N} \int_{0}^{t} s^{N-1} m(s) d s\right)^{1 /(p-1)} d t
$$

is the unique positive solution of the following problem:

$$
\begin{aligned}
\operatorname{div}\left(|\nabla z|^{p-2} \nabla z\right)= & -\left(\frac{\gamma-p+1}{p-1}\right)^{p-1} m(r), \quad x \in \mathbb{R}^{N}, r=\|x\|, \\
& z \longrightarrow 0, \quad|x| \longrightarrow \infty
\end{aligned}
$$


We claim that $v_{k}^{(p-1-\gamma) /(p-1)} \leq z$ on $|x| \leq k$. Clearly, this inequality holds for $|x|=k$, where $v_{k}^{(p-1-\gamma) /(p-1)}=0$. Letting $g=v_{k}^{(p-1-\gamma) /(p-1)}$ gives

$$
\begin{aligned}
\operatorname{div}\left(|\nabla g|^{p-2} \nabla g\right) \geq & -\left(\frac{\gamma-p+1}{p-1}\right)^{p-1} m(r) \\
& +\left(\frac{\gamma-p+1}{p-1}\right)^{p-1} \gamma v_{k}^{-\gamma-1}\left|\nabla v_{k}\right|^{p}
\end{aligned}
$$

Thus

$$
-\operatorname{div}\left(|\nabla g|^{p-2} \nabla g\right) \leq-\operatorname{div}\left(|\nabla z|^{p-2} \nabla z\right) \quad \text { in }|x|<k
$$

and from Lemma 2.1, we obtain $g=v_{k}^{(p-1-\gamma) /(p-1)} \leq z$ if $|x| \leq k$. Let $w=$ $(1 / z)^{(p-1) /(\gamma-p+1)}$ and note that $v_{k} \geq w$ in $\mathbb{R}^{N}$. Consequently, $v \geq w$ in $\mathbb{R}^{N}$ and (A) is proved. Since $w \rightarrow \infty$ as $|x| \rightarrow \infty$, it is clear that (B) follows easily from (A).

LEMmA 2.5. Suppose that $p-1<\alpha \leq \beta, \beta>q-1$, and $p, q>1$. The problem

$$
\begin{aligned}
& \operatorname{div}\left(|\nabla g|^{p-2} \nabla g\right)=m(|x|) g^{\alpha}+n(|x|) g^{\beta}, \\
& \operatorname{div}\left(|\nabla h|^{q-2} \nabla h\right)=m(|x|) h^{\alpha}+n(|x|) h^{\beta}
\end{aligned}
$$

has an entire explosive positive radial solution provided that the $C\left(\mathbb{R}^{N}\right)$ functions $m(x), n(x) \geq C>0$ and satisfy

$$
\begin{aligned}
& \int_{0}^{\infty}\left(t^{1-N} \int_{0}^{t} s^{N-1} m(s) d s\right)^{1 /(p-1)} d t<\infty, \\
& \int_{0}^{\infty}\left(t^{1-N} \int_{0}^{t} s^{N-1} n(s) d s\right)^{1 /(q-1)} d t<\infty .
\end{aligned}
$$

Proof. From Lemma 2.3, for each natural number $k$, let $v_{k}$ be a positive solution of the boundary value problem

$$
\begin{aligned}
\operatorname{div}\left(\left|\nabla v_{k}\right|^{p-2} \nabla v_{k}\right) & =m(|x|) v_{k}^{\alpha}+n(|x|) v_{k}^{\beta}, \quad|x|<k, \\
v_{k} & \rightarrow \infty, \quad|x| \longrightarrow k .
\end{aligned}
$$

Again, by the maximum principle, we can show that

$$
v_{1} \geq v_{2} \geq \cdots \geq v_{k} \geq v_{k+1} \geq \cdots>0
$$

in $\mathbb{R}^{N}$. To complete the proof, it is sufficient to show that there exists a function $w \in C\left(\mathbb{R}^{N}\right)$ such that $w \rightarrow \infty$ as $|x| \rightarrow \infty$ and $v_{k} \geq w$ in $\mathbb{R}^{N}$ for all $k$. To do this, we first consider the equation

$$
\operatorname{div}\left(|\nabla u|^{p-2} \nabla u\right)=[m(|x|)+n(|x|)] u^{\beta}
$$


By Lemma 2.4 , (2.48) has a positive solution $u$ on $\mathbb{R}^{N}$ such that $u(x) \rightarrow \infty$ as $|x| \rightarrow \infty$. We claim that $w=u-1$ is a desired lower boundary for $v_{k}$. Indeed, since

$$
\begin{aligned}
\operatorname{div}\left(\left|\nabla\left(v_{k}+1\right)\right|^{p-2} \nabla\left(v_{k}+1\right)\right) & =\operatorname{div}\left(\left|\nabla v_{k}\right|^{p-2} \nabla v_{k}\right)=m v_{k}^{\alpha}+n v_{k}^{\beta} \\
& \leq m\left(v_{k}+1\right)^{\alpha}+n\left(v_{k}+1\right)^{\beta} \\
& \leq(m+n)\left(v_{k}+1\right)^{\beta} \text { for }|x|>k,
\end{aligned}
$$

and clearly $v_{k}+1>u$ as $|x| \rightarrow k$, Lemma 2.1 implies that $v_{k}+1 \geq u$ for $|x| \leq k$. Hence, $v=\lim _{k \rightarrow \infty} v_{k} \geq u-1$ on $\mathbb{R}^{N}$. Again, by the standard regularity argument for elliptic problems, it is a straightforward argument to prove that $v$ is the desired solution of (2.43). By a similar argument, we can show that (2.44) has an entire explosive positive radial solution.

LEMMA 2.6. Suppose that $g_{R}$ and $h_{R}$ are positive radial solutions of the problem

$$
\begin{gathered}
\operatorname{div}\left(\left|\nabla g_{R}\right|^{p-2} \nabla g_{R}\right)=m(r) g_{R}^{\alpha}+n(r) g_{R}^{\beta}, \quad 0 \leq r<R, \\
g_{R}(r) \longrightarrow \infty, \quad r \longrightarrow R^{-}, \\
\operatorname{div}\left(\left|\nabla h_{R}\right|^{q-2} \nabla h_{R}\right)=m(r) h_{R}^{\alpha}+n(r) h_{R}^{\beta}, \quad 0 \leq r<R, \\
h_{R}(r) \longrightarrow \infty, \quad r \longrightarrow R^{-},
\end{gathered}
$$

where $m$ and $n$ are nonnegative $C([0, \infty))$ functions and $p-1<\alpha \leq \beta, \beta>$ $q-1, p>1$, and $q>1$. Then $\lim _{R \rightarrow 0^{+}} g_{R}(0)=\infty$ and $\lim _{R \rightarrow 0^{+}} h_{R}(0)=\infty$.

Proof. Since $g_{R}^{\prime}(r) \geq 0$ and $m, n$ are bounded on $[0,1]$, we get

$$
\begin{aligned}
\left(g_{R}^{\prime}(r)\right)^{p-1} & =r^{1-N} \int_{0}^{r} s^{N-1}\left[m(s) g_{R}^{\alpha}(s)+n(s) g_{R}^{\beta}(s)\right] d s \\
& \leq \int_{0}^{r}\left[m(s) g_{R}^{\alpha}(s)+n(s) g_{R}^{\beta}(s)\right] d s \\
& \leq a g_{R}^{\alpha}(r)+b g_{R}^{\beta}(r),
\end{aligned}
$$

then

$$
\begin{aligned}
g_{R}^{\prime}(r) & \leq\left(a g_{R}^{\alpha}(r)+b g_{R}^{\beta}(r)\right)^{1 /(p-1)} \\
& \leq \begin{cases}a^{1 /(p-1)} g_{R}^{\alpha /(p-1)}(r)+b^{1 /(p-1)} g_{R}^{\beta /(p-1)}, & \text { for } p \geq 2, \\
2^{(2-p) /(p-1)}\left(a^{1 /(p-1)} g_{R}^{\alpha /(p-1)}(r)+b^{1 /(p-1)} g_{R}^{\beta /(p-1)}\right), & \text { for } 1<p<2,\end{cases}
\end{aligned}
$$

where

$$
a=\int_{0}^{1} m(s) d s, \quad b=\int_{0}^{1} n(s) d s .
$$


Thus we have

$$
\begin{gathered}
\frac{g_{R}^{\prime}(r)}{a^{1 /(p-1)} g_{R}^{\alpha /(p-1)}(r)+b^{1 /(p-1)} g_{R}^{\beta /(p-1)}} \leq 1 \quad \text { for } p \geq 2, \\
\frac{g_{R}^{\prime}(r)}{2^{(2-p) /(p-1)}\left(a^{1 /(p-1)} g_{R}^{\alpha /(p-1)}(r)+b^{1 /(p-1)} g_{R}^{\beta /(p-1)}\right)} \leq 1 \quad \text { for } 1<p<2,
\end{gathered}
$$

which implies that

$$
\begin{gathered}
-\frac{d}{d r} \int_{g_{R}(r)}^{\infty} \frac{d s}{a^{1 /(p-1)} s^{\alpha /(p-1)}+b^{1 /(p-1)} s^{\beta /(p-1)}} \leq 1 \quad \text { for } p \geq 2, \\
-\frac{d}{d r} \int_{g_{R}(r)}^{\infty} \frac{d s}{2^{(2-p) /(p-1)}\left(a^{1 /(p-1)} s^{\alpha /(p-1)}+b^{1 /(p-1)} s^{\beta /(p-1)}\right)} \leq 1 \quad \text { for } 1<p<2 .
\end{gathered}
$$

Now, integrating from 0 to $R$ and recalling that $g_{R}(r) \rightarrow \infty$ as $r \rightarrow R^{-}$, we get

$$
\begin{gathered}
\int_{g_{R}(0)}^{\infty} \frac{d s}{a^{1 /(p-1)} s^{\alpha /(p-1)}+b^{1 /(p-1)} s^{\beta /(p-1)}} \leq R \quad \text { for } p \geq 2, \\
\int_{g_{R}(0)}^{\infty} \frac{d s}{2^{(2-p) /(p-1)}\left(a^{1 /(p-1)} s^{\alpha /(p-1)}+b^{1 /(p-1)} s^{\beta /(p-1)}\right)} \leq R \quad \text { for } 1<p<2 .
\end{gathered}
$$

Letting $R \rightarrow 0^{+}$yields

$$
\lim _{R \rightarrow 0^{+}} \int_{g_{R}(0)}^{\infty} \frac{d s}{a^{1 /(p-1)} s^{\alpha /(p-1)}+b^{1 /(p-1)} s^{\beta /(p-1)}}=0 .
$$

Hence, we have $g_{R}(0) \rightarrow \infty$ as $R \rightarrow 0^{+}$. By a similar argument, we can show that $h_{R}(0) \rightarrow \infty$ as $R \rightarrow 0^{+}$.

LEMMA 2.7. Let $g, h$ be any entire explosive positive radial solutions of (2.43), (2.44) given in Lemma 2.5 and define the sequences $\left\{u_{k}\right\}$ and $\left\{v_{k}\right\}$ by

$$
\begin{aligned}
& u_{k}(r)=a+\int_{0}^{r}\left(t^{1-N} \int_{0}^{t} s^{N-1} m(s) v_{k-1}^{\alpha}(s) d s\right)^{1 /(p-1)} d t, \quad r \geq 0, \\
& v_{k}(r)=b+\int_{0}^{r}\left(t^{1-N} \int_{0}^{t} s^{N-1} n(s) u_{k-1}^{\beta}(s) d s\right)^{1 /(q-1)} d t, \quad r \geq 0,
\end{aligned}
$$

where $u_{0}=a, 0 \leq a \leq \min \{g(0), h(0)\}$, and $v_{0}(r)=b, 0 \leq b \leq \min \{g(0), h(0)\}$. Then

(a) $u_{k}(r) \leq u_{k+1}(r) ; v_{k}(r) \leq v_{k+1}(r), r \in \mathbb{R}^{+}, k \geq 1$;

(b) $u_{k}(r) \leq g(r)$ and $v_{k}(r) \leq h(r), r \in \mathbb{R}^{+}, k \geq 1$.

Thus $\left\{u_{k}\right\}$ and $\left\{v_{k}\right\}$ converge and the limit functions are entire positive radial solutions of system (1.4). 
Proof. (a) Obviously, $v_{0}<v_{1}$. This then yields $u_{1}<u_{2}$ by (2.58). Consequently, $v_{1}<v_{2}$ by (2.59), which yields $u_{2}<u_{3}$ by (2.58). Continuing this line of reasoning, we obtain that the sequences $\left\{u_{k}\right\}$ and $\left\{v_{k}\right\}$ are monotonically increasing.

(b) We note first that, since $g$ is radial, we get

$$
g(r)=g(0)+\int_{0}^{r}\left(t^{1-N} \int_{0}^{t} s^{N-1}\left[m(s) g^{\alpha}(s)+n(s) g^{\beta}(s)\right] d s\right)^{1 /(p-1)} d t .
$$

Now, it is clear (since $g^{\prime}(r) \geq 0$ ) that $b=v_{0} \leq g(0) \leq g(r)$ for all $r \geq 0$. Thus we have

$$
\begin{aligned}
u_{1}(r) & \leq a+\int_{0}^{r}\left(t^{1-N} \int_{0}^{t} s^{N-1} m(s) g^{\alpha}(s) d s\right)^{1 /(p-1)} d t \\
& \leq g(0)+\int_{0}^{r}\left(t^{1-N} \int_{0}^{t} s^{N-1}\left[m(s) g^{\alpha}(s)+n(s) g^{\beta}(s)\right] d s\right)^{1 /(p-1)} d t=g(r) .
\end{aligned}
$$

Thus we have $u_{1} \leq g$. Similar arguments will show, in sequence, that $v_{1} \leq h$, $u_{2} \leq g, \ldots$

3. Main results. By a modification of the method given in [13], we establish the following results.

THEOREM 3.1. Suppose that $m$ and $n$ are $C\left(\mathbb{R}^{N}\right)$ functions and $m(x)=$ $m(|x|), n(x)=n(|x|) \geq C>0,0<\alpha \leq p-1$, and $0<\alpha \leq \beta \leq q-1$. Then there exists an entire positive radial solution of (1.4) with any central values

$$
u(0)=a \geq 0, \quad v(0)=b \geq 0 .
$$

If, in addition, the functions $m$ and $n$ satisfy

$$
\begin{aligned}
& \int_{0}^{\infty}\left(t^{1-N} \int_{0}^{t} s^{N-1} m(s) d s\right)^{1 /(p-1)} d t=\infty \\
& \int_{0}^{\infty}\left(t^{1-N} \int_{0}^{t} s^{N-1} n(s) d s\right)^{1 /(q-1)} d t=\infty
\end{aligned}
$$

then all entire positive radial solutions of (1.4) are large solutions. On the other hand, if $m$ and $n$ satisfy

$$
\begin{aligned}
& \int_{0}^{\infty}\left(t^{1-N} \int_{0}^{t} s^{N-1} m(s) d s\right)^{1 /(p-1)} d t<\infty, \\
& \int_{0}^{\infty}\left(t^{1-N} \int_{0}^{t} s^{N-1} n(s) d s\right)^{1 /(q-1)} d t<\infty,
\end{aligned}
$$

then all entire positive radial solutions of (1.4) are bounded. 
PROof. Note that radial solutions of (1.4) are solutions of the ordinary differential equation system

$$
\begin{aligned}
& \left(\Phi_{p}\left(u^{\prime}\right)\right)^{\prime}+\frac{N-1}{r} \Phi_{p}\left(u^{\prime}\right)=m(r) v^{\alpha}, \\
& \left(\Phi_{q}\left(v^{\prime}\right)\right)^{\prime}+\frac{N-1}{r} \Phi_{q}\left(v^{\prime}\right)=n(r) u^{\beta} .
\end{aligned}
$$

Thus solutions of (1.4) are simply solutions of

$$
\begin{aligned}
& u(r)=a+\int_{0}^{r}\left(t^{1-N} \int_{0}^{t} s^{N-1} m(s) v^{\alpha}(s) d s\right)^{1 /(p-1)} d t, \quad r \geq 0, \\
& v(r)=b+\int_{0}^{r}\left(t^{1-N} \int_{0}^{t} s^{N-1} n(s) u^{\beta}(s) d s\right)^{1 /(q-1)} d t, \quad r \geq 0 .
\end{aligned}
$$

Let $\left\{u_{k}\right\}$ and $\left\{v_{k}\right\}$ be as defined in Lemma 2.7, where the central values $(a, b)$ may be any ordered pair of nonnegative numbers. We will show that the monotonically increasing sequences $\left\{u_{k}\right\}$ and $\left\{v_{k}\right\}$ are bounded above whenever $r$ is bounded and hence converge on $\mathbb{R}^{N}$. Indeed, we note that, since $v_{k}^{\prime}(r) \geq 0$,

$$
\begin{aligned}
u_{k}(r) & \leq u_{k+1}(r) \leq a+v_{k}^{\alpha /(p-1)}(r) \int_{0}^{r}\left(t^{1-N} \int_{0}^{t} s^{N-1} m(s) d s\right)^{1 /(p-1)} d t \\
& =a+v_{k}^{\alpha /(p-1)}(r) f(r),
\end{aligned}
$$

where

$$
f(r)=\int_{0}^{r}\left(t^{1-N} \int_{0}^{t} s^{N-1} m(s) d s\right)^{1 /(p-1)} d t
$$

Similarly, we get $v_{k}(r) \leq b+u_{k}^{\beta /(q-1)}(r) g(r)$, where

$$
g(r)=\int_{0}^{r}\left(t^{1-N} \int_{0}^{t} s^{N-1} n(s) d s\right)^{1 /(q-1)} d t
$$

Combining these, we get

$$
u_{k}(r) \leq a+f(r)\left(b+u_{k}^{\beta /(q-1)}(r) g(r)\right)^{\alpha /(p-1)} .
$$

Since $0<\alpha<p-1$, we know that $(c+d)^{\alpha /(p-1)} \leq c^{\alpha /(p-1)}+d^{\alpha /(p-1)}$ for any nonnegative constants $c$ and $d$. Therefore, by applying this inequality, we get

$$
\begin{aligned}
u_{k}(r) & \leq a+f(r)\left(b^{\alpha /(p-1)}+u_{k}^{\alpha \beta /(p-1)(q-1)} g^{\alpha /(p-1)}\right) \\
& =c(r)+u_{k}^{\alpha \beta /(p-1)(q-1)} h(r) .
\end{aligned}
$$

If $\alpha \beta<(p-1)(q-1)$, using the elementary inequality (see [19, page 30])

$$
x^{1 / m} y^{1 / n} \leq \frac{x}{m}+\frac{y}{n}
$$


where $x \geq 0, y \geq 0$, and $1 / m+1 / n=1$ with $m>1$, we observe that

$$
u_{k}(r) \leq c(r)+\frac{\alpha \beta}{(p-1)(q-1)} u_{k}+\left(1-\frac{\alpha \beta}{(p-1)(q-1)}\right) h^{1 /(1-\alpha \beta /(p-1)(q-1))} .
$$

Thus

$$
u_{k}(r) \leq \frac{c(r)+(1-\alpha \beta /(p-1)(q-1)) h^{1 /(1-\alpha \beta /(p-1)(q-1))}}{1-\alpha \beta /(p-1)(q-1)} \quad \forall k .
$$

Similarly, there exists a function $\psi$ such that $v_{k}(r) \leq \psi(r)$ for all $k$ if $\alpha \beta<$ $(p-1)(q-1)$. On the other hand, if $\alpha \beta=(p-1)(q-1)$ so that $\alpha=p-1$ and $\beta=q-1$, we substitute (3.6) into the equation for $v_{k}$ to get

$$
v_{k}(r) \leq b+\int_{0}^{r}\left(t^{1-N} \int_{0}^{t} s^{N-1} q(s)\left[a+f(s) v_{k}(s)\right]^{q-1} d s\right)^{1 /(q-1)} d t
$$

We consider two cases here: (i) $q \geq 2$ and (ii) $1<q<2$.

In case (i), we have that $q-1 \geq 1$. Using the inequality $(1+x)^{q-1} \leq 2^{q-2}(1+$ $x^{q-1}$ ) for $x \geq 0$, we have

$$
\begin{aligned}
v_{k}(r) \leq & b+\int_{0}^{r} 2^{(q-2) /(q-1)}\left(t^{1-N} \int_{0}^{t} s^{N-1}\left[a^{q-1}+v^{q-1} f^{q-1}(s)\right] q(s) d s\right)^{1 /(q-1)} d t \\
\leq & b+\int_{0}^{r} 2^{(q-2) /(q-1)}\left[t^{1-N} \int_{0}^{t} s^{N-1} a^{q-1} q(s) d s\right. \\
& \left.\quad+t^{1-N} \int_{0}^{t} s^{N-1} v^{q-1}(s) f^{q-1}(s) q(s) d s\right]^{1 /(q-1)} d t \\
\leq & \bar{b}(r) \\
& +2^{(q-2) /(q-1)} \int_{0}^{r} t^{(1-N) /(q-1)}\left(\int_{0}^{t} s^{N-1} v^{q-1} f^{q-1}(s) q(s) d s\right)^{1 /(q-1)} d t \\
\leq & \bar{b}(r)+2^{(q-2) /(q-1)} \int_{0}^{r} v_{k}(t)\left(\int_{0}^{t}\left(\frac{s}{t}\right)^{N-1} f^{q-1}(s) q(s) d s\right)^{q-1} d t .
\end{aligned}
$$

Letting $a(r)=\int_{0}^{r} v_{k}(t)\left(\int_{0}^{t}(s / t)^{N-1} f^{q-1}(s) q(s) d s\right)^{q-1} d t$, then

$$
a^{\prime}(r)=v_{k}(r)\left(\int_{0}^{r}\left(\frac{s}{r}\right)^{N-1} f^{q-1}(s) q(s) d s\right)^{1 /(q-1)} .
$$

Therefore,

$$
\begin{aligned}
a^{\prime}(r) \leq & \bar{b}(r)\left(\int_{0}^{r}\left(\frac{s}{r}\right)^{N-1} f^{q-1}(s) q(s) d s\right)^{1 /(q-1)} \\
& +2^{(q-2) /(q-1)} a(r)\left(\int_{0}^{r}\left(\frac{s}{r}\right)^{N-1} f^{q-1}(s) q(s) d s\right)^{1 /(q-1)} .
\end{aligned}
$$


Letting

$$
\begin{aligned}
d^{\prime}(r) & =2^{(q-2) /(q-1)}\left(\int_{0}^{r}\left(\frac{s}{r}\right)^{N-1} f^{q-1}(s) q(s) d s\right)^{1 /(q-1)}, \\
c(r) & =\bar{b}(r)\left(\int_{0}^{r}\left(\frac{s}{r}\right)^{N-1} f^{q-1}(s) q(s) d s\right)^{1 /(q-1)},
\end{aligned}
$$

then

$$
\left(e^{-d(r)} a(r)\right)^{\prime} \leq c(r) e^{-d(r)} \Longleftrightarrow a(r) \leq K(r)
$$

Thus

$$
v_{k}(r) \leq \bar{c}(r)
$$

Similarly, there exists a function $\bar{d}(r)$ such that $u_{k}(r) \leq \bar{d}(r)$ for all $k$ if $\alpha=$ $p-1$. Thus the sequences $\left\{u_{k}\right\}$ and $\left\{v_{k}\right\}$ are bounded above on bounded sets and therefore converge. Let $u(r)=\lim _{k \rightarrow \infty} u_{k}(r)$ and $v(r)=\lim _{k \rightarrow \infty} v_{k}(r)$. By standard elliptic regularity theory, it can be shown that $(u, v)$ is the desired solution of (1.4).

In case (ii), we have that $0<q-1<1$ using the inequality $(1+x)^{q-1} \leq$ $1+x^{q-1}$ for $x \geq 0$. Similarly, as in (i), there exist functions $\bar{c}(r)$ and $\bar{d}(r)$ such that $u_{k}(r) \leq \bar{c}(r)$ and $v_{k}(r) \leq \bar{d}(r)$ for all $k$ if $\alpha=p-1$ and $\beta=q-1$.

If (3.2) hold and $(u, v)$ is a positive solution of (3.5), then, clearly,

$$
u(r) \geq a+b^{\alpha /(p-1)} f(r),
$$

and, similarly, $v(r) \geq b+a^{\beta /(q-1)} g(r)$. However, $\lim _{r \rightarrow \infty} f(r)=\lim _{r \rightarrow \infty} g(r)=$ $\infty$. Thus, $(u, v)$ is an entire explosive radial solution.

On the other hand, if the inequalities (3.3) hold, then $\lim _{r \rightarrow \infty} f(r)<\infty$ and $\lim _{r \rightarrow \infty} g(r)<\infty$ so that the estimates above providing upper bounds for the sequences $\left\{u_{k}\right\}$ and $\left\{v_{k}\right\}$ may be chosen independent of $r$ so that the solution $(u, v)$ is bounded above (and, in fact, any solution of (3.5) will be bounded when the inequalities (3.3) hold).

We now give our main theorems for the superlinear case, where $p-1<\alpha \leq$ $\beta, \beta \geq q-1$, and $2 \leq q \leq p$. We use the notation $\mathbb{R}^{+}=[0, \infty)$ and define the set $G$ as

$$
\begin{aligned}
G=\left\{(a, b) \in \mathbb{R}^{+} \times \mathbb{R}^{+} \mid u(0)=a, v(0)=b,\right. \\
\\
(u, v) \text { is an entire radial solution of }(1.4)\} .
\end{aligned}
$$

THEOREM 3.2. There are infinitely many entire positive radial solutions of system (1.4) provided that the $C\left(\mathbb{R}^{N}\right)$ functions $m$ and $n$ satisfy (3.3). The set $G$ is a closed bounded convex subset of $\mathbb{R}^{+} \times \mathbb{R}^{+}$. Furthermore, the set $G$ satisfies

$$
T \subset G \subset R,
$$


where the triangle $T$ and the rectangle $R$ are given by

$$
T=\left\{(u, v) \in \mathbb{R}^{+} \times \mathbb{R}^{+} \mid \frac{u}{A}+\frac{v}{B} \leq 1\right\}, \quad R=[0, A] \times[0, B],
$$

in which $A=\sup \left\{a \in \mathbb{R}^{+} \mid(a, 0) \in G\right\}$ and $B=\sup \left\{b \in \mathbb{R}^{+} \mid(0, b) \in G\right\}$.

Proof. From Lemma 2.7, it is clear that $[0, g(0)] \times[0, h(0)] \subset G$ so that $G$ is nonempty. We will show that $G$ is a bounded, closed, and convex set and then prove relationship (3.23).

As a preliminary, note that, if $(a, b) \in G$, then any pair $\left(a_{0}, b_{0}\right)$ for which $0 \leq a_{0} \leq a$ and $0 \leq b_{0} \leq b$ must be in $G$ since the process used in Lemma 2.7 can be repeated with

$$
\begin{aligned}
& u_{k}(r)=a_{0}+\int_{0}^{r}\left(t^{1-N} \int_{0}^{t} s^{N-1} m(s) v_{k-1}^{\alpha}(s) d s\right)^{1 /(p-1)} d t \\
& v_{k}(r)=b_{0}+\int_{0}^{r}\left(t^{1-N} \int_{0}^{t} s^{N-1} n(s) u_{k-1}^{\beta}(s) d s\right)^{1 /(q-1)} d t,
\end{aligned}
$$

and $v_{0}=b, u_{0}=a$. Then, as in Lemma 2.7, the sequences $\left\{u_{k}\right\}$ and $\left\{v_{k}\right\}$ are monotonically increasing. Then, letting $(U, V)$ be the solution of (3.5) with central values $(a, b)$, we can easily prove, since $b_{0} \leq b$, that $v_{0} \leq V$. Thus, $u_{1} \leq U$ (since, also, $a_{0} \leq a$ ), and consequently $v_{1} \leq V$, and so on. Hence, we get $u_{k} \leq U$ and $v_{k} \leq V$, and therefore, $u \leq U$ and $v \leq V$, where $(u, v)=\lim _{k \rightarrow \infty}\left(u_{k}, v_{k}\right)$ is a solution of (1.4) (with central values $\left(a_{0}, b_{0}\right)$ ).

To prove that $G$ is bounded, assume that it is not. Therefore, since $[0, a] \times$ $[0, b] \subset G$ whenever $(a, b) \in G$, we must have either $[0, \infty) \times\{0\} \subset G$ or $\{0\} \times$ $[0, \infty) \subset G$. Without loss of generality, we assume that $[0, \infty) \times\{0\} \subset G$. Let $\bar{m}(r)=\min \{m(r), n(r)\}$ and let $h$ be a positive radial solution of

$$
\begin{gathered}
\operatorname{div}\left(|\nabla h|^{p-2} \nabla h\right)=2^{-\alpha} \bar{m}(r) h^{\alpha}, \quad 0 \leq r \leq 1, \\
h(r) \longrightarrow \infty, \quad r \longrightarrow 1^{-} .
\end{gathered}
$$

(See Lemma 2.3 for the proof of existence.) Let $(u, v)$ be any solution, which exists by hypothesis, to (3.5) with $a>h(0)$ and $b=0$. Without loss of generality, we will assume that $a \geq 1$. We now show that $h \leq u+v$ for all $r \geq 0$ which, if proven, will contradict the fact that $u+v$ exists for all $r \geq 0$. Clearly, $h(0)<a \leq u(0)+v(0)$. Thus there exists $\epsilon>0$ such that $h(r)<u(r)+v(r)$ for all $r \in[0, \epsilon)$. Let

$$
R_{0}=\sup \{\epsilon>0 \mid h(r)<u(r)+v(r), \forall r \in[0, \epsilon)\}
$$


We claim that $R_{0}=1$. Indeed, suppose that $R_{0}<1$. Since $h(r)<u(r)+v(r)$ in $\left[0, R_{0}\right)$, elementary estimates yield

$$
\begin{aligned}
h\left(R_{0}\right)= & h(0)+\int_{0}^{R_{0}}\left(t^{1-N} \int_{0}^{t} s^{N-1} 2^{-\alpha} \bar{m}(s) h^{\alpha}(s) d s\right)^{1 /(p-1)} d t \\
< & h(0)+\int_{0}^{R_{0}}\left(t^{1-N} \int_{0}^{t} s^{N-1} 2^{-\alpha} \bar{m}(s)[u+v]^{\alpha}(s) d s\right)^{1 /(p-1)} d t \\
\leq & h(0)+\int_{0}^{R_{0}}\left(t^{1-N} \int_{0}^{t} s^{N-1} \bar{m}(s)\left(u^{\alpha}(s)+v^{\alpha}(s)\right) d s\right)^{1 /(p-1)} d t \\
\leq & h(0)+\int_{0}^{R_{0}}\left(t^{1-N} \int_{0}^{t} s^{N-1}\left(m v^{\alpha}(s)+n u^{\alpha}(s)\right) d s\right)^{1 /(p-1)} d t \\
\leq & h(0)+\int_{0}^{R_{0}}\left(t^{1-N} \int_{0}^{t} s^{N-1}\left(m v^{\alpha}(s)+n u^{\beta}(s)\right) d s\right)^{1 /(p-1)} d t \\
< & a+\int_{0}^{R_{0}}\left(t^{1-N} \int_{0}^{t} s^{N-1} m v^{\alpha}(s) d s\right)^{1 /(p-1)} d t \\
& +\int_{0}^{R_{0}}\left(t^{1-N} \int_{0}^{t} s^{N-1} n u^{\beta}(s) d s\right)^{1 /(q-1)} d t \\
< & u\left(R_{0}\right)+v\left(R_{0}\right) .
\end{aligned}
$$

Thus, since $h\left(R_{0}\right)<u\left(R_{0}\right)+v\left(R_{0}\right)$, there exists $\delta>0$ such that $h(r)<u(r)+$ $v(r)$ in $\left[0, R_{0}+\delta\right)$. This contradicts the fact that $R_{0}$ is a supremum. Thus, $R_{0}=1$, establishing the boundedness of the set $G$.

To prove that $G$ is closed, we let $\left(a_{0}, b_{0}\right) \in \partial G$ and show that $\left(a_{0}, b_{0}\right) \in G$. Let $(u, v)$ be the solution of (3.5) which corresponds to $a=a_{0}$ and $b=b_{0}$. Without loss of generality, we may assume that $\max \left\{a_{0}, b_{0}\right\}>K=g(0)$, where the function $g$ is given in Lemma 2.7. If $\max \left\{a_{0}, b_{0}\right\}=a_{0}$, then $K \leq a_{0}-1 / n$ for large $n$ so that $u_{n}(r) \geq K$ for all $r \geq 0$ and for all $n$ sufficiently large where

$$
\begin{aligned}
& u_{n}=a_{0}-\frac{1}{n}+\int_{0}^{r}\left(t^{1-N} \int_{0}^{t} s^{N-1} m(s) v_{n-1}^{\alpha}(s) d s\right)^{1 /(p-1)} d t \\
& v_{n}=b_{0}+\int_{0}^{r}\left(t^{1-N} \int_{0}^{t} s^{N-1} n(s) u_{n-1}^{\beta}(s) d s\right)^{1 /(q-1)} d t
\end{aligned}
$$

Thus we note that

$$
\begin{gathered}
m v_{n}^{\alpha} \geq \bar{m}(r) v_{n}^{\alpha}, \\
n u_{n}^{\beta} \geq n u_{n}^{\beta-\alpha} u_{n}^{\alpha} \geq n K^{\beta-\alpha} u_{n}^{\alpha} \geq \bar{m}(r) u_{n}^{\alpha},
\end{gathered}
$$

where $\bar{m}(r)=\min \left\{1, K^{\beta-\alpha}\right\} \min \{m(r), n(r)\}$. Thus

$$
\begin{aligned}
& \operatorname{div}\left(\left|\nabla u_{n}\right|^{p-2} \nabla u_{n}\right) \geq \bar{m}(r) v_{n}^{\alpha}, \\
& \operatorname{div}\left(\left|\nabla v_{n}\right|^{q-2} \nabla v_{n}\right) \geq \bar{m}(r) u_{n}^{\alpha} .
\end{aligned}
$$


Let $h_{1}(r)$ and $h_{2}(r)$ be positive solutions of

$$
\begin{gathered}
\operatorname{div}\left(\left|\nabla h_{1}\right|^{p-2} \nabla h_{1}\right)=\bar{m}(r) h_{1}^{\alpha}, \quad 0 \leq r<R_{0}, \\
h_{1}(r) \longrightarrow \infty, \quad r \longrightarrow R_{0}^{-}, \\
\operatorname{div}\left(\left|\nabla h_{2}\right|^{q-2} \nabla h_{2}\right)=\bar{m}(r) h_{2}^{\alpha}, \quad 0 \leq r<R_{0}, \\
h_{2}(r) \longrightarrow \infty, \quad r \longrightarrow R_{0}^{-},
\end{gathered}
$$

where $R_{0}$ is an arbitrary positive real number. It is now easy to show by Lemma 2.1 that $u_{n} \leq h_{1}$ and $v_{n} \leq h_{2}$; thus $u_{n}+v_{n} \leq h_{1}+h_{2}$ in $\left[0, R_{0}\right]$. Hence, $u+v=$ $\lim _{n \rightarrow \infty}\left(u_{n}+v_{n}\right) \leq h_{1}+h_{2}$ on $\left[0, R_{0}\right]$. Since $R_{0}$ is arbitrary, the functions $u$ and $v$ exist on $\mathbb{R}^{N}$ and are hence entire so that $\left(a_{0}, b_{0}\right) \in G$. On the other hand, if $\max \left\{a_{0}, b_{0}\right\}=b_{0}$, then $K \leq b_{0}-1 / n$ for large $n$ so that $v_{n} \geq K$ for all $r \geq 0$ and for all sufficiently large $n$. Then $u_{n}(r) \geq K^{\alpha} f(r)$, where $f(r)=\int_{0}^{r}\left(t^{1-N} \int_{0}^{t} s^{N-1} m(s) d s\right)^{1 /(p-1)} d t$, and the proof continues as before with $K$ replaced by $K^{\alpha} f(r)$.

To prove that $G$ is convex, suppose that $(a, b) \in G$ and $(\bar{a}, \bar{b}) \in G$. Let $\lambda \in$ $(0,1)$, let $(u, v)$ be the solution of (3.5), and let $(U, V)$ be the solution of (3.5) when $(a, b)$ is replaced by $(\bar{a}, \bar{b})$. We need to prove that $\lambda(a, b)+(1-\lambda)(\bar{a}, \bar{b}) \in$ $G$. To do this, we let $\left\{u_{n}\right\},\left\{v_{n}\right\},\left\{U_{n}\right\}$, and $\left\{V_{n}\right\}$ be the increasing sequences of functions, as developed in Lemma 2.7, such that $u_{n} \nearrow u, v_{n} \nearrow v, U_{n} \nearrow U$, and $V_{n}>V$. Likewise, let $\left\{w_{n}\right\}$ and $\left\{z_{n}\right\}$ be the sequences developed again as in Lemma 2.7 corresponding to central values $\lambda a+(1-\lambda) \bar{a}$ and $\lambda b+(1-\lambda) \bar{b}$, respectively. We also let $z_{0}=\lambda b+(1-\lambda) \bar{b}$. We will show that the increasing sequences $\left\{w_{n}\right\}$ and $\left\{z_{n}\right\}$ satisfy

$$
\begin{gathered}
w_{n} \leq \lambda u_{n}+(1-\lambda) U_{n}, \\
z_{n} \leq \lambda v_{n}+(1-\lambda) V_{n},
\end{gathered}
$$

which, in turn, implies that $\left\{w_{n}\right\}$ and $\left\{z_{n}\right\}$ converge, and hence, their limits are entire, giving $\lambda(a, b)+(1-\lambda)(\bar{a}, \bar{b}) \in G$. Clearly, $z_{0} \leq \lambda v_{0}+(1-\lambda) V_{0}$. Then

$$
\begin{aligned}
w_{1}(r) & =\lambda a+(1-\lambda) \bar{a}+\int_{0}^{r}\left(t^{1-N} \int_{0}^{t} s^{N-1} m(s) z_{0}^{\alpha}(s) d s\right)^{1 /(p-1)} d t \\
& \leq \lambda a+(1-\lambda) \bar{a}+\int_{0}^{r}\left(t^{1-N} \int_{0}^{t} s^{N-1} m(s)\left[\lambda v_{0}+(1-\lambda) V_{0}\right]^{\alpha}(s)\right)^{1 /(p-1)} d t
\end{aligned}
$$

Since $\alpha>p-1 \geq 1$, however, we know that $(\lambda c+(1-\lambda) d)^{\alpha} \leq \lambda c^{\alpha}+(1-\lambda) d^{\alpha}$ for any nonnegative numbers $c$ and $d$. Applying this inequality, we get

$$
\begin{aligned}
w_{1}(r) \leq & \lambda a+(1-\lambda) \bar{a} \\
& +\int_{0}^{r}\left(t^{1-N} \int_{0}^{t} s^{N-1} m(s)\left[\lambda v_{0}^{\alpha}+(1-\lambda) V_{0}^{\alpha}\right](s) d s\right)^{1 /(p-1)} d t \\
= & \lambda u_{1}+(1-\lambda) U_{1} .
\end{aligned}
$$


Using this result, we can prove similarly that $z_{1} \leq \lambda v_{1}+(1-\lambda) V_{1}$ which, in turn, can be used to get $w_{2} \leq \lambda u_{2}+(1-\lambda) U_{2}$. Continuing this process will produce (3.33).

To prove (3.23), it is clear that, since $(A, 0)$ and $(0, B)$ are in $G$ and $G$ is convex, the line $x / A+y / B=1$ is in $G$. And, as noted earlier, if $(a, b) \in G$, then $\left(x_{0}, y_{0}\right) \in G$ whenever $0 \leq x_{0} \leq a$ and $0 \leq y_{0} \leq b$. Hence, $T \subset G$. Similarly, $G \subset R$ for if $\left(a_{0}, b_{0}\right) \in G$, then $\left(a_{0}, 0\right) \in G$ and $\left(0, b_{0}\right) \in G$. Thus $0 \leq a_{0} \leq A$ and $0 \leq b_{0} \leq B$ so that $\left(a_{0}, b_{0}\right) \in \mathbb{R}$. This completes the proof.

THEOREM 3.3. Let $E(G)$ be the closure of the set $\{(a, b) \in \partial G \mid a>0, b>0\}$. Any entire positive radial solution $(u, v)$ of (1.4) with central value $(u(0), v(0))$ $\in E(G)$ is an explosive solution.

Proof. Let $u_{n}$ and $v_{n}$ be defined as positive solutions of

$$
\begin{aligned}
& u_{n}=u(0)+\frac{1}{n}+\int_{0}^{r}\left(t^{1-N} \int_{0}^{t} m(s) v_{n}^{\alpha}(s) d s\right)^{1 /(p-1)} d t \\
& v_{n}=v(0)+\frac{1}{n}+\int_{0}^{r}\left(t^{1-N} \int_{0}^{t} n(s) u_{n}^{\beta}(s) d s\right)^{1 /(q-1)} d t
\end{aligned}
$$

where $(u(0), v(0)) \in E(G)$. We note that $u_{n}^{\prime}(r) \geq 0$ and $v_{n}^{\prime}(r) \geq 0$. Also, since $(u(0)+1 / n, v(0)+1 / n) \notin G$, for each $n=1,2,3, \ldots$, there exists $R_{n}<\infty$ such that

$$
\begin{gathered}
\lim _{r \rightarrow R_{n}^{-}} u_{n}(r)=\infty, \quad \lim _{r \rightarrow R_{n}^{-}} v_{n}(r)=\infty, \\
R_{1} \leq R_{2} \leq R_{3} \leq \cdots .
\end{gathered}
$$

From (3.36) and the fact that $v_{n}^{\prime} \geq 0$, we get

$$
v_{n}(r) \leq v(0)+\frac{1}{n}+u_{n}^{\beta /(q-1)}(r) \int_{0}^{\infty}\left(t^{1-N} \int_{0}^{t} s^{N-1} n(s) d s\right)^{1 /(q-1)} d t
$$

which implies that

$$
v_{n}(r) \leq C u_{n}(r)+K u_{n}^{\beta /(q-1)}(r),
$$

where $C$ is any upper bound on $(v(0)+1 / n) /(u(0)+1 / n)$ and

$$
K=\int_{0}^{\infty}\left(t^{1-N} \int_{0}^{t} s^{N-1} n(s) d s\right)^{1 /(q-1)} d t<\infty .
$$

Since $u_{n}$ satisfies

$$
\operatorname{div}\left(|\nabla u|^{p-2} \nabla u\right)=m v_{n}^{\alpha}
$$

we use (3.39) to get

$$
\operatorname{div}\left(|\nabla u|^{p-2} \nabla u\right) \leq m f\left(u_{n}\right)
$$


where $f(s)=\left(C s+K s^{\beta /(q-1)}\right)^{\alpha}$. Define

$$
F(s)=\int_{s}^{\infty} \frac{d t}{f^{1 /(p-1)}(t)},
$$

which is well defined for $s>0$ since $\alpha>p-1$. Note also that

$$
F^{\prime}(s)=-\frac{1}{f^{1 /(p-1)}(s)}, \quad F^{\prime \prime}(s)=\frac{f^{\prime}(s)}{(p-1)\left(f^{1 /(p-1)}\right)^{p}}>0 .
$$

Thus

$$
\begin{aligned}
\operatorname{div}\left(\left|\nabla F\left(u_{n}\right)\right|^{p-2} \nabla F\left(u_{n}\right)\right)= & -\left|F^{\prime}\left(u_{n}\right)\right|^{p-1} \operatorname{div}\left(\left|\nabla u_{n}\right|^{p-2} \nabla u_{n}\right) \\
& +(p-1)\left|F^{\prime}\left(u_{n}\right)\right|^{p-2} F^{\prime \prime}\left(u_{n}\right)\left|\nabla u_{n}\right|^{p} \\
\geq & -\left|F^{\prime}\left(u_{n}\right)\right|^{p-1} m f\left(u_{n}\right)=-m .
\end{aligned}
$$

Hence,

$$
\operatorname{div}\left(\left|\nabla F\left(u_{n}\right)\right|^{p-2} \nabla F\left(u_{n}\right)\right) \geq-m(r)
$$

or

$$
\left(r^{N-1}\left|F^{\prime}\left(u_{n}\right)\right|^{p-2} F^{\prime}\left(u_{n}\right)\right)^{\prime} \geq-r^{N-1} m(r) .
$$

Integrate this over $[0, r]$ where $0<r<R_{n}$ to get

$$
\frac{d}{d r} F\left(u_{n}\right) \geq-\left(r^{1-N} \int_{0}^{r} s^{N-1} m(s) d s\right)^{1 /(p-1)} .
$$

Now integrate this over $\left[r, R_{n}\right]$. Noting that $u_{n}(r) \rightarrow \infty$ as $r \rightarrow R_{n}^{-}$(and hence, $F\left(u_{n}(r)\right) \rightarrow 0$ as $r \rightarrow R_{n}^{-}$), we get

$$
-F\left(u_{n}(r)\right) \geq-\int_{r}^{R_{n}}\left(s^{1-N} \int_{0}^{s} t^{N-1} m(t) d t\right)^{1 /(p-1)} d s .
$$

Thus we have

$$
F\left(u_{n}\right) \leq \int_{r}^{R_{n}}\left(s^{1-N} \int_{0}^{s} t^{N-1} m(t) d t\right)^{1 /(p-1)} d s .
$$

Since $F^{\prime}(s)<0$ for $s>0$, we have

$$
u_{n}(r) \geq F^{-1}\left(\int_{r}^{R_{n}}\left(s^{1-N} \int_{0}^{s} t^{N-1} m(t) d t\right)^{1 /(p-1)} d s\right) .
$$

Now, let $n \rightarrow \infty$ so that $R_{n} \rightarrow R \leq \infty$ and $u_{n} \rightarrow u$ to produce

$$
u_{n}(r) \geq F^{-1}\left(\int_{r}^{R}\left(s^{1-N} \int_{0}^{s} t^{N-1} m(t) d t\right)^{1 /(p-1)} d s\right), \quad 0 \leq r<R .
$$


Letting $r \rightarrow R^{-}$and since from Lemma 2.6,

$$
\lim _{s \rightarrow 0^{+}} F^{-1}(s)=\infty,
$$

we have

$$
\lim _{r \rightarrow R^{-}} u(r) \geq \lim _{r \rightarrow R} F^{-1}\left(\int_{r}^{R}\left(s^{1-N} \int_{0}^{s} t^{N-1} m(t) d t\right)^{1 /(p-1)} d s\right)=\infty .
$$

Since $u$ is entire, we conclude that $R=\infty$ and $\lim _{r \rightarrow \infty} u(r)=\infty$. This completes the proof.

ACKNOWLEDgments. The author thanks Professors A. V. Lair and A. W. Wood for bringing to attention the boundary explosive problems for quasilinear elliptic systems. The author also thanks the referee for many valuable suggestions and comments.

\section{REFERENCES}

[1] V. Anuradha, C. Brown, and R. Shivaji, Explosive nonnegative solutions to two-point boundary value problems, Nonlinear Anal. 26 (1996), no. 3, 613-630.

[2] S. Chen and G. Lu, Existence and nonexistence of positive radial solutions for a class of semilinear elliptic system, Nonlinear Anal., Ser. A: Theory Methods 38 (1999), no. 7, 919-932.

[3] K.-S. Cheng and W.-M. Ni, On the structure of the conformal scalar curvature equation on $\mathbb{R}^{n}$, Indiana Univ. Math. J. 41 (1992), no. 1, 261-278.

[4] Ph. Clément, D. G. de Figueiredo, and E. Mitidieri, Positive solutions of semilinear elliptic systems, Comm. Partial Differential Equations 17 (1992), no. 5-6, 923-940.

[5] Ph. Clément, R. F. Manásevich, and E. Mitidieri, Positive solutions for a quasilinear system via blow up, Comm. Partial Differential Equations 18 (1993), no. 12, 2071-2106.

[6] G. Díaz and R. Letelier, Explosive solutions of quasilinear elliptic equations: existence and uniqueness, Nonlinear Anal. 20 (1993), no. 2, 97-125.

[7] P. L. Felmer, R. F. Manásevich, and F. de Thélin, Existence and uniqueness of positive solutions for certain quasilinear elliptic systems, Comm. Partial Differential Equations 17 (1992), no. 11-12, 2013-2029.

[8] Z. M. Guo, Some existence and multiplicity results for a class of quasilinear elliptic eigenvalue problems, Nonlinear Anal. 18 (1992), no. 10, 957-971.

[9]___ Existence of positive radial solutions for a class of quasilinear elliptic systems in annular domains, Chinese J. Contemp. Math. 17 (1996), no. 4, 337350 (Chinese).

[10] _ On the existence of positive solutions for a class of semilinear elliptic systems, J. Partial Differential Equations 10 (1997), no. 3, 193-212.

[11] A. V. Lair and A. W. Wood, Large solutions of semilinear elliptic equations with nonlinear gradient terms, Int. J. Math. Math. Sci. 22 (1999), no. 4, 869-883.

[12] _ L L Large solutions of semilinear elliptic problems, Nonlinear Anal., Ser. A: Theory Methods 37 (1999), no. 6, 805-812.

[13] _ Existence of entire large positive solutions of semilinear elliptic systems, J. Differential Equations 164 (2000), no. 2, 380-394. 
[14] _ Large solutions of sublinear elliptic equations, Nonlinear Anal., Ser. A: Theory Methods 39 (2000), no. 6, 745-753.

[15] A. C. Lazer and P. J. McKenna, On a problem of Bieberbach and Rademacher, Nonlinear Anal. 21 (1993), no. 5, 327-335.

[16] E. Mitidieri, A Rellich type identity and applications, Comm. Partial Differential Equations 18 (1993), no. 1-2, 125-151.

[17] _ Nonexistence of positive solutions of semilinear elliptic systems in $\mathbf{R}^{N}$, Differential Integral Equations 9 (1996), no. 3, 465-479.

[18] E. Mitidieri, G. Sweers, and R. C. A. M van der Vorst, Non-existence theorems for systems of quasilinear partial differential equations, Differential Integral Equations 8 (1995), no. 6, 1331-1354.

[19] D. S. Mitrinović, Analytic Inequalities, Die Grundlehren der Mathematischen Wisenschaften, vol. 1965, Springer-Verlag, New York, 1970.

[20] L. A. Peletier and R. C. A. M. van der Vorst, Existence and nonexistence of positive solutions of nonlinear elliptic systems and the biharmonic equation, Differential Integral Equations 5 (1992), no. 4, 747-767.

[21] S. I. Pohožaev, The Dirichlet problem for the equation $\Delta u=u^{2}$, Soviet Math. Dokl. 1 (1960), 1143-1146 (Russian).

[22] J. Serrin and H. Zou, Nonexistence of positive solutions of Lane-Emden systems, Differential Integral Equations 9 (1996), no. 4, 635-653.

[23] _ Existence of positive entire solutions of elliptic Hamiltonian systems, Comm. Partial Differential Equations 23 (1998), no. 3-4, 577-599.

[24] R. C. A. M. van der Vorst, Variational identities and applications to differential systems, Arch. Rational Mech. Anal. 116 (1991), no. 4, 375-398.

[25] S.-H. Wang, Existence and multiplicity of boundary blow-up nonnegative solutions to two-point boundary value problems, Nonlinear Anal., Ser. A: Theory Methods 42 (2000), no. 2, 139-162.

[26] Z. Yang and Q. Lu, Nonexistence of positive radial solutions for a class of quasilinear elliptic system, Commun. Nonlinear Sci. Numer. Simul. 5 (2000), no. 4, 184-187.

[27] _ Blow-up estimates for a non-Newtonian filtration system, Appl. Math. Mech. 22 (2001), no. 3, 287-294.

[28] _ Blow-up estimates for a quasilinear reaction-diffusion system, Math. Methods Appl. Sci. 26 (2003), no. 12, 1005-1023.

Yang Zuodong: School of Mathematics and Computer Science, Nanjing Normal University, Nanjing, Jiangsu 210097, China

E-mail address: zdyang_jin@263.net 


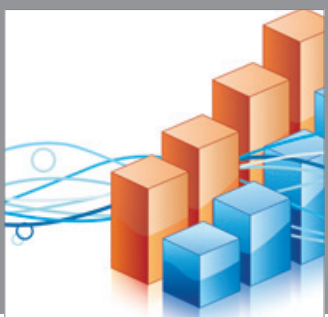

Advances in

Operations Research

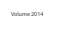

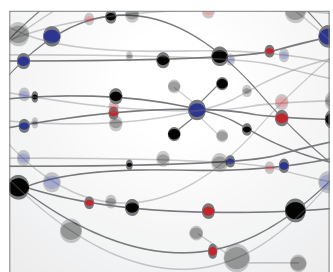

\section{The Scientific} World Journal
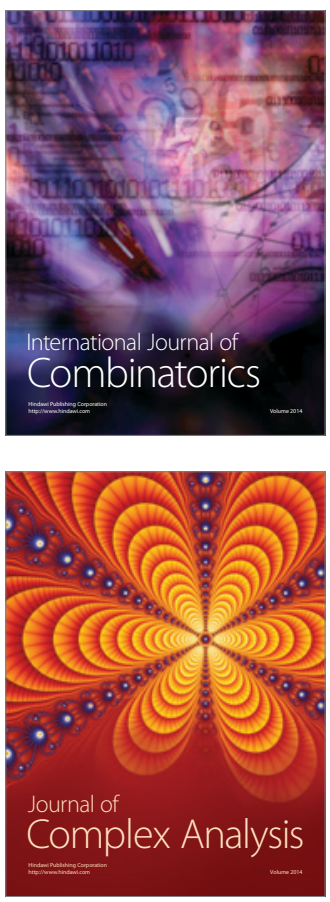

International Journal of

Mathematics and

Mathematical

Sciences
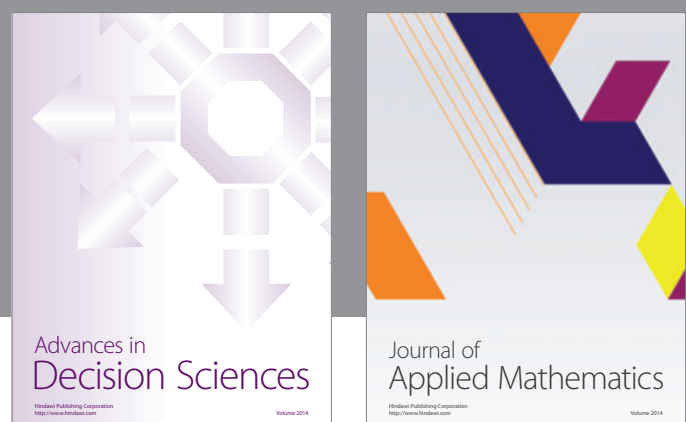

Journal of

Applied Mathematics
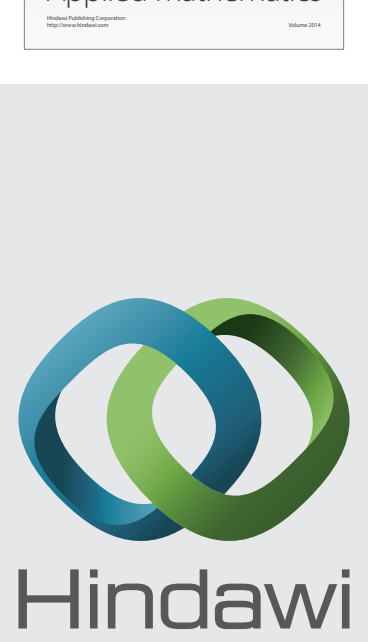

Submit your manuscripts at http://www.hindawi.com
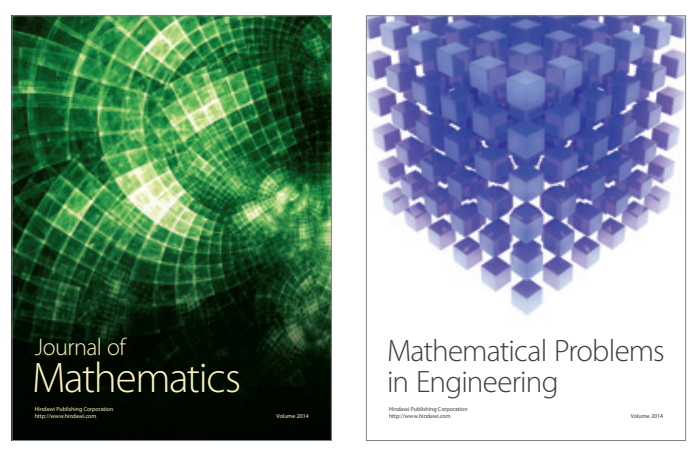

Mathematical Problems in Engineering
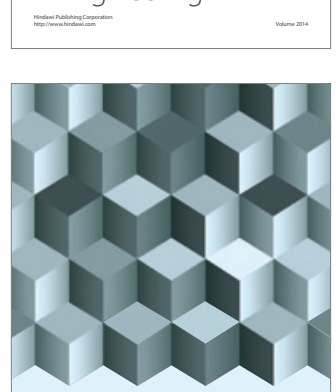

Journal of

Function Spaces
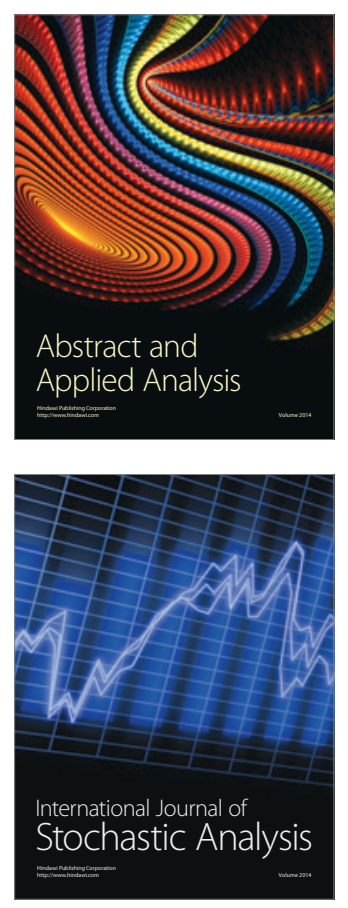

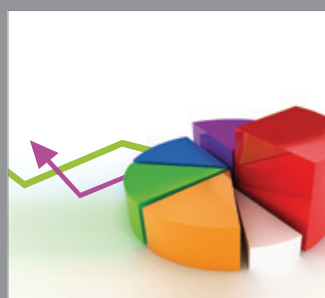

ournal of

Probability and Statistics

Promensencen
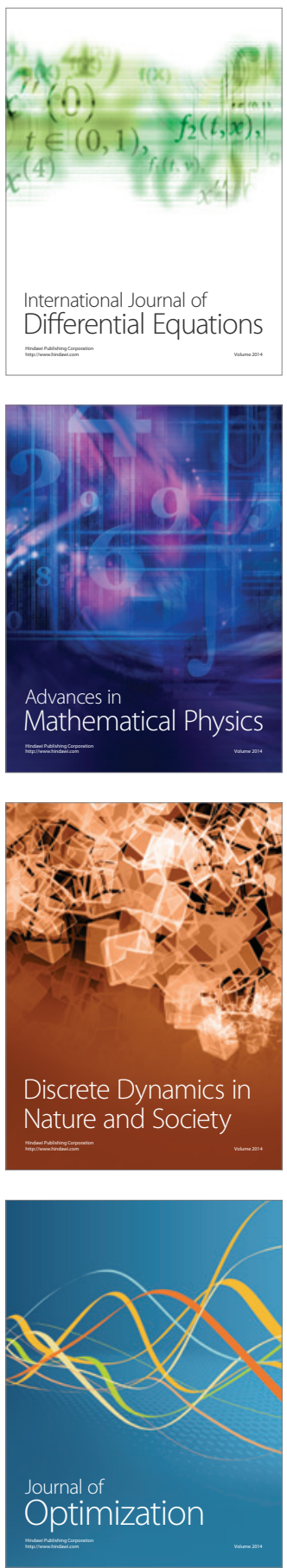